\title{
Design of a new type of safety protection device for gas bottle cabinet
}

\author{
Lianghui Guo ${ }^{1, *}$ and Wenting $\mathrm{Jia}^{2}$ \\ ${ }^{1}$ College of Safety Science and Engineering, Xinjiang Institute of Engineering, Urumchi, China \\ ${ }^{2}$ Asset Management Service, Xinjiang Institute of Engineering, Urumchi, China
}

\begin{abstract}
Keywords: GSM voice, Short message control technology, Gas bottle cabinet, Monitor, Alarm concentration.

Abstract. Aiming at the problem of explosion caused by leakage of flammable and explosive gas cylinder, a new type of safety protection device for gas bottle cabinet is designed to solve this problem. The design of this new type of gas bottle cabinet safety protection device is to use GSM voice, short message control technology, sensor technology, wifi technology, plc control technology and so on, to monitor the gas in the gas bottle cabinet in real time. Once the gas in the cylinder leaks and the concentration of the leakage gas reaches the set alarm concentration, the alarm will occur, and the exhaust fan will start to discharge the leakage gas and send an alarm message to the pre-set mobile phone at the same time.
\end{abstract}

\section{Introduction}

Today, with the rapid development of science and technology, many scientific research and experimental teaching work are completed in the laboratory. Toxic, harmful, flammable and explosive gases are often used. Due to poor management, explosion and poisoning accidents may be caused. In recent years, many explosion and poisoning accidents have occurred in universities and scientific research institutions, so the management of the laboratory is becoming more and more important. On April 5, 2015, a gas explosion occurred in a laboratory of the school of chemical engineering, China University of mining and technology, which injured 5 people, one of whom died due to ineffective rescue[1]. On December 18, 2015, an explosion occurred in the chemical laboratory of Tsinghua University, resulting in the death of a postdoctoral student. After the explosion, experts analyzed that the explosion was caused by hydrogen leakage, and the high temperature generated by the explosion further led to the combustion of combustible materials in the laboratory [2]. On September 21, 2016, an explosion occurred in a laboratory of the school of chemistry, chemical engineering and bioengineering of Donghua University located in Songjiang University Park, causing serious injury to two students [3]. Wei Bing, Zheng Yue, et al. Studied the explosion of gas cylinder in laboratory[4,5]. Fu Jing et al. Took 107 university

${ }^{*}$ Corresponding author:313512418@qq.com 
laboratory accidents from 2001 to 2016 as samples for statistical analysis, studied the inherent factors that led to laboratory accidents, and pointed out that the triggering factors such as the failure and defect of equipment and its protective devices were taken as the key points of university laboratory safety supervision [6]. Zhao Jing et al. Established a fire and explosion model of combustible gas based on fault tree according to the case of fire and explosion in chemical laboratory[7]. At present, most of the management of the gas cylinder cabinet in the laboratory adopts the independent individual management mode, that is, when a gas cylinder leaks, only the gas cylinder cabinet alarms and exhausts, and requires the laboratory personnel to be present to find out. If the experimenter is not on the spot, it is difficult to find the leakage. Even if the experimenter is on the spot, some gases are colorless, tasteless, flammable and explosive. If the alarm on the gas cylinder cabinet fails, it is difficult for the experimenter to find the gas leakage. When the gas concentration reaches the limit value, it will lead to explosion and personnel poisoning accidents. This management method of gas cylinder can not meet the requirements of laboratory safety management. Therefore, it is necessary to study the design of a new type of safety protection device for gas cylinder cabinet.

\section{Laboratory hazardous gas management system}

\subsection{GSM voice and SMS control system}

When the gas leakage in the laboratory exceeds the limit, GSM voice and short message control is adopted, which is mainly aimed at the networked management of inflammable, explosive, poisonous and harmful gas in the laboratory's individual equipment place, and the automatic operation is adopted. By means of GSM voice and short message control, communication and data transmission are carried out for the leakage of inflammable and explosive gases and toxic and harmful gases, timely notice management personnel and make data backup, and start emergency plan at the same time. The system can effectively prevent accidents caused by toxic and harmful gas leakage. On the one hand, the anti-riot solenoid valve is activated by the gas controller to automatically close the main valve of the gas source to control the further spread of the gas hazard. On the other hand, the exhaust fan is automatically started to discharge the leaking toxic and harmful gas into the outdoor atmosphere to prevent dangerous energy accumulation. Through gas signal of the controller, start the GSM voice, SMS control, notify the preset manager's phone number, in the form of text messages to inform management personnel to the scene of the accident in time for further processing, controlled by GSM voice, SMS and Wifi to real-time data backup, to provide data support for preventive measures.

\subsection{Design of new gas cabinet safety device}

Part of the new design of the gas cabinet by a safety guard gas cabinet, a ventilation fan, the main components of GSM voice/SMS alarm controller, Wifi module controller, solenoid valves and the gas explosion-proof filters and other components, new gas cabinet structure shown in figure 1 safety apparatus shown in figure. 


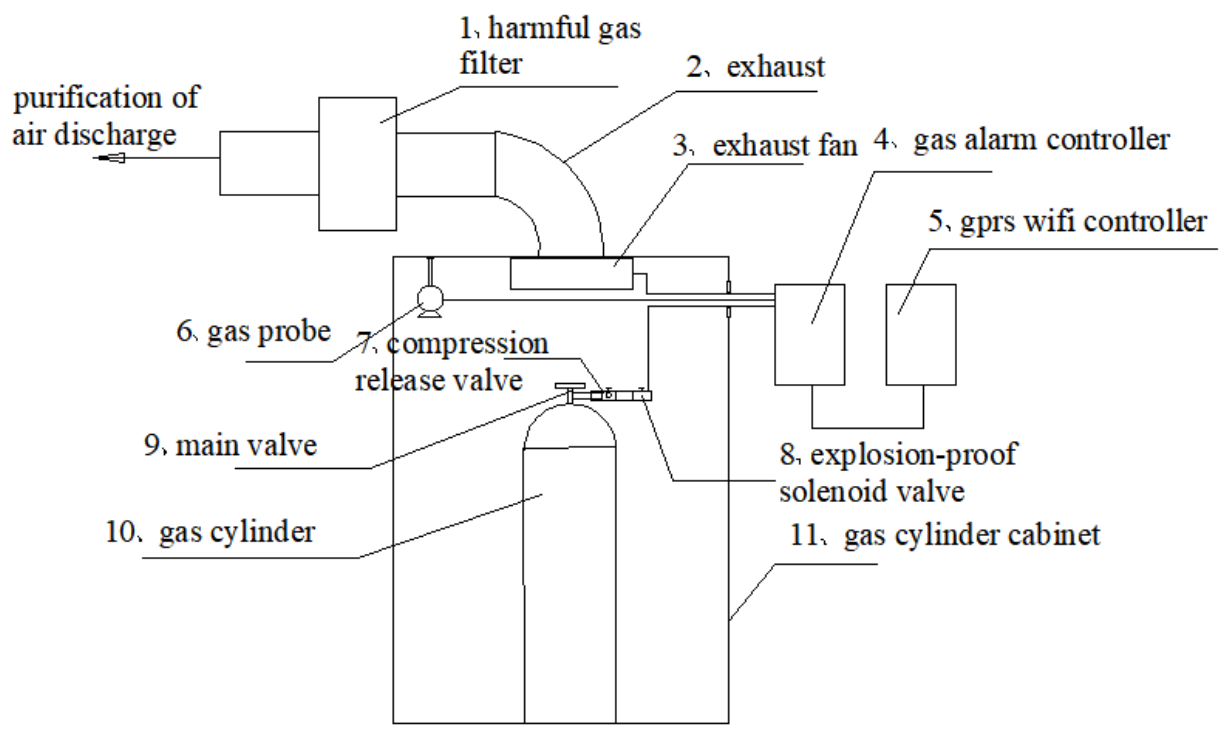

Fig. 1. Safety protection assembly diagram of new gas cylinder cabinet.

\section{Application of the safety protection device of the new gas cylinder cabinet}

\subsection{The operation process of the safety protection device of the new gas cylinder cabinet}

First open the gas main valve and then open the pressure reducing valve during the normal use of the cylinder. If the toxic, harmful, flammable and explosive gas in the cylinder leaks, the flammable and explosive gas probe instantly senses the gas, and the gas alarm controller gives the GSM module a high level signal at the same time. The output signal starts the relay, starts the exhaust fan, and shuts off the total gas circuit solenoid valve at the same time. The GSM controller can transmit the set data and information to the preset mobile phone number, and the management personnel can carry on the remote management to avoid the expansion of the accident. in the network transmission mode, the serial port equipment of all the cylinder cabinets in the laboratory are incorporated into the system, and the cylinder leakage in any cylinder cabinet can send data to the designated server on the network through this module. The module can also accept the data from the server and forward the data information to the serial port equipment, which enables managers to control the solenoid valve remotely through the network and transmit the data to the PC through Wifi to achieve intelligent management. The solenoid valve shuts off the gas source of the total gas path and eliminates the constant leakage of the gas source. If the leaked gas is toxic, harmful, flammable and explosive, it can be filtered through a filter to ensure that the gas discharged into the atmosphere is a filtered pure gas. At the same time, the system can also be conveniently incorporated into the monitoring and monitoring system through the communication port to achieve network management. The operation principle of the new gas cylinder cabinet safety protection device is shown in figure 2 . 


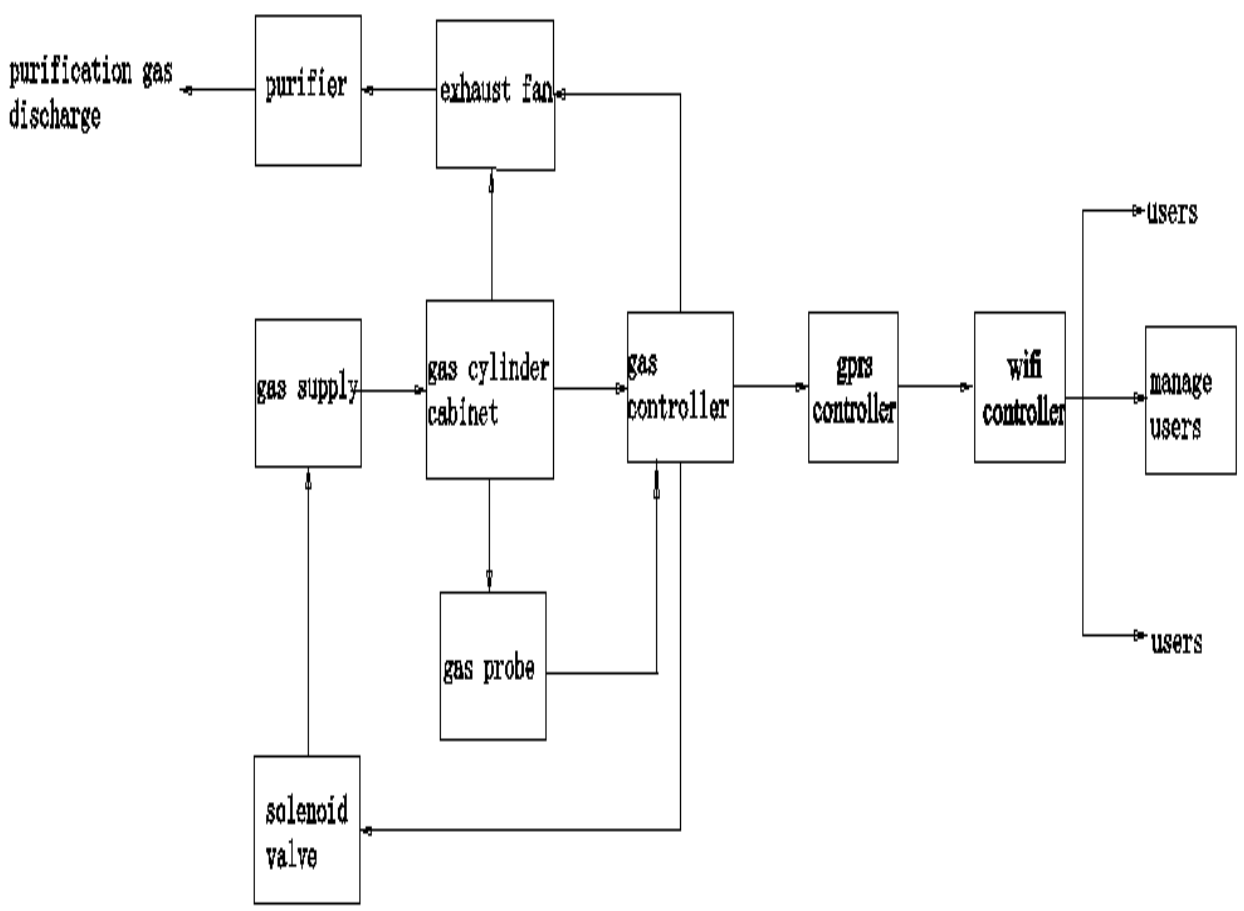

Fig. 2. Operation schematic diagram of a new type of gas cylinder cabinet safety protection device.

\subsection{New operating parameters of the gas cabinet safety device}

With methane gas laboratory for example, methane gas leak detection for laboratory gas alarm cabinet. Methane gas concentration sensoris set warning value of $1 \%$ when the methane gasleaks, the gas concentration of $1 \%$ the alarmstarts alarm, while the exhaust fan is turned on, the total off gas circuit. Alarm control system architecture shown in figure 3.

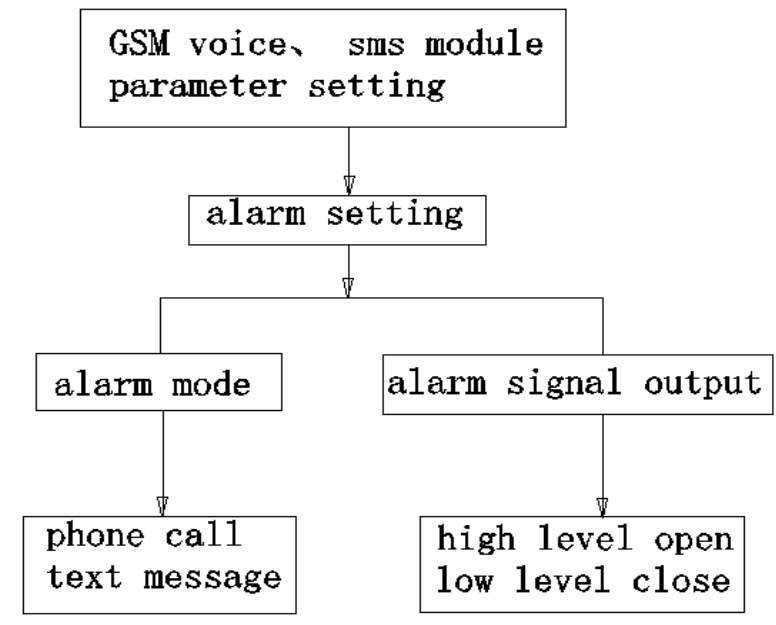

Fig. 3. Setting structure diagram of alarm control system. 


\section{Summary}

On the management of poisonous and harmful gas and inflammable and explosive gas, the on-line dynamic monitoring and testing technology is applied to form the network management of the monitoring of the gas cylinder cabinet in the laboratory. If any gas cylinder or instrument in the cabinet is leaking, the gas sensor can quickly feed back the signal to the GSM controller and send an alarm signal when the gas is turned off. Send a short message of alarm to the mobile phone of the management personnel to rush to the scene for processing. The system realizes on-line dynamic monitoring and real-time control of inflammable and explosive gases and effectively avoids dangerous accidents caused by gas leakage.

\section{References}

1. Liu Qingxiang, Li Weihao. Five students were killed and 4 injured in an explosion in the first laboratory of China Mining University [N] Hyundai KuaiBao, 2015-04-06

2. Liu Mu, Zhou Dan, Lei Jia. Tsinghua Laboratory explosion 32-year-old postdoctoral death [N] Beijing Youth Daily, 2015-12-19

3. Zhang Xiangmei, Cheng Xinru, Yu Meng, etc. Donghua University Laboratory explosion 3 students injured [N] Beijing Youth Daily, 2016-09-22

4. Wei Bing. Common accidents and elimination methods in chemical laboratory [J] Inner Mongolia Petrochemical Industry, 2011 (10): 137-138

5. Zheng Yue. First aid and emergency treatment of common accidents in chemical analysis laboratory [J] Guangzhou Chemical Industry, 2014 (22): 232-234

6. Fu Jing, Liu Hong, Liu Wenbo. Cause analysis and management countermeasures of fire and explosion accidents in colleges and universities [J]. Journal of Jilin Institute of Chemical Technology, 2018, 35 (05): 87 -92.

7. Zhao Jing, Zhang Fuqun. Study on fire and explosion risk in chemical laboratory based on fault tree analysis [J]. Liaoning Chemical Industry, 2018, 47 (05): 453-455+458. 